\title{
The Time-Frequency Characters Analysis Of The Snoring Signal
}

\author{
Yinhong Zhang ${ }^{1,2}$, Quanlu $\mathrm{Li}^{1}$, Jing $\mathrm{Wu}^{2}$, Jin $\mathbf{L i}^{2}$ \\ ${ }^{1}$ Applied Acoustics Institute, Shaanxi Normal University, Xi'an, 710062, China \\ ${ }^{2}$ College of Physics and Information Technology, Shaanxi Normal University, Xi'an, 710062, China \\ zhangyh@snnu.edu.cn
}

\begin{abstract}
The snoring is a typical inspiratory sound appearing during sleep. It is an important characters of upper airway obstruction. The severe snoring sound leads to the Obstructive Sleep Apnea Syndrome during persistent ventilatory movements and it can result in cessation of breathing. This paper presents a time-frequency characters analysis method of non-stationary snoring signal that based on the discrete wavelet transform. The experimental result shows that the characters of different time-frequency domain of the snoring signal and offers value for analyzing the temporal feature of snoring sound in health medical treatment.

Index Terms - Discrete wavelet transform; Nocturnal snoring; Time-frequency analysis; MATLAB
\end{abstract}

\section{I . Introduction}

Snoring is defined as the sounds made by vibration in the sift palate and their adjacent tissues (such as the posterior faucal pillars) during sleep. Researchers have shown that it is the most important symptom connected with the Obstructive Sleep Apnea Syndrome(OSAS), as well as the cause of much disruption to bed partners and to the snorer. Severe snoring can result in sleep-related upper airway narrowing, which leads to respiratory flow limitation and increased respiratory effort. Strong inspiratory suction may secondarily, cause a total upper airway blocked. The OSAS is a highly prevalent disease in which upper airways are collapsed during sleep, leading to serious consequences. Recently, several studies concerning to prevalence of snoring and sleep apnea have been published[1]. The OSAS has important clinical implications, ranging from disruption of sleep with daytime sequel of excessive sleepiness to suspected cardiovascular consequences in the long term[2]. Fourier transform, short-time Fourier transform, cestrum method, the bilateral spectral method, autoregressive spectral estimation, maximum entropy spectral estimation, wavelet transform methods are usually used in signal analysis. As time-frequency signal's analysis methods offer simultaneous interpretation of the signal in both time and frequency which allows local, transients or intermittent components to be elucidated[3,4]. The wavelet transform is proposed as a more exact method because it gives the possibility to focus on the signal spectral characteristics. Therefore, it can express signals with different frequency components, especially those mutational signals. It is discussed the analysis method of snoring signal that based on orthogonal wavelet transform in this paper. Its time-frequency domain characteristics are showed and the upper respiratory tract the extent of obstruction caused by snoring sound pathological signals are diagnosed accurately.

\section{II . Wavelet Transform Theories}

Wavelet transforms can be used to project the data features into different levels of time resolution. Wavelet analysis of a signal is capable of providing both the time and frequency content of the signal simultaneously, hence giving a time-frequency representation. Wavelet use a variable size windowing technique. Long time windows provide precise low frequency information and shorter time windows provide high frequency information. The discrete wavelet transform (DWT) is used for developing the multi-resolution fusion technique. The DWT chooses scales and time based on powers of two; i.e., the dyadic scales and time.

The DWT analyzes the signal at different frequency bands with different resolutions by decomposing the signal into coarse approximation and into detail information. The DWT employs two sets of functions as a wavelet orthonormal basis for this decomposition. They are called scaling functions, and wavelet function $w_{i, k}[n]$. These functions are associated with quadrature mirror lowpass filters $h[n]$ and highpass filters $g[n]$, respectively.

$$
\begin{aligned}
& \phi_{i, k}[n]=2^{i / 2} h\left[2^{i} n-k\right] \\
& w_{i, k}[n]=2^{i / 2} g\left[2^{i} n-k\right]
\end{aligned}
$$

Where $i$ and $k$ are scale and translation parameters, respectively. A discrete signal $x[n]$ can be fully-reconstructed by these scaling function and wavelet function according to the decomposition:

$$
x[n]=\sum_{k \in z} a[N, k] \phi_{N, k}[n]+\sum_{i=1}^{N} \sum_{k \in z} d[i, k] w_{i, k}[n]
$$

$i$ is the increasing level index, $N$ is maximum decomposition level, and $k$ is time shift. $a[N, k]$ and $d[i, k]$ are approximation coefficients, respectively. They can be obtained by inner product:

$$
\begin{aligned}
& a[N, k]=\left\langle x[n], \phi_{N, k}[n]\right\rangle \\
& d[i, k]=\left\langle x[n], w_{i, k}[n]\right\rangle
\end{aligned}
$$


After DWT to the signal, we get an approximation coefficient $a[N, k]$ and a series of wavelet coefficients $d[i, k][5]$.

Although defined as an integral transform, the DWT is usually implemented to use a dyadic filter bank where the filter coefficients are directly derived from the wavelet function used in the analysis. The sampled snoring signal is putting into the filter.

\section{III. snoring signal analysis}

The spectral characteristics of volumes are an important parameter to the signal. Time-domain waveform and power spectral analysis methods are used. Snoring sound is typical non-stationary signal and includes many repeated complex waveforms. The waveforms of snore signal are varied from different people, its spectral characteristics are changing from time to time. They reported that a greater sound intensity appeared to be the result of greater negative pressures on resumption of breathing in apneic snorers, resulting in high flow rates, turbulent flow and greater forces on the vibrating structures. The power spectrum of snoring signal is obtained from analyzing the spectral of them. which are located in the beginning from $100 \mathrm{~Hz}$, up to $1200 \mathrm{~Hz}$ frequency range [6].

The waveform and frequency spectrum of the mutational snore signal depend primarily on obstruction of breath. The greater degree of obstruction, the more narrow band of the signal, its spectrum of low frequencies which are less than $200 \mathrm{~Hz}$ would be filtered out, vice versa. Therefore, many spectral analysis methods can not clearly analyze the indefinite variability snore. Wavelet transform have the capacity of local features of signals in the time domain and frequency domain. For high-frequency components of signals which are more than $500 \mathrm{~Hz}$, using gradually sharp temporal resolution analysis in order to observe the signals immediately, For low-frequency components of signals using gradually a sharp frequency resolution analysis method in order to move into a much slower observed signal points (the total change in trend), so it is suitable for analyzing the temporal feature of snoring sound signal.

\section{Experimental apparatus and signal analysis}

\section{A. Hardware design for collecting snoring signal}

Azadeh Yadollahi et al. have recorded snoring sound signals from two microphones simultaneously: one placed over trachea (tracheal microphone) and one placed over the forehead of the patient (ambient microphone). An automatic method was developed to classify breath and snore sound segments based on their energy, zero crossing rate and formants of the sound signals. The effects of body and neck position on the classification's performance were also investigated in that study[7]. Michael Herzog et al. have recorded snoring sounds in a quiet room. The examination under simulated snoring and the recording of snoring sounds were peformed in an upright, $45^{\circ}$ and supine position. Snoring was recorded by a microphone (ECM-2000,Monacor, Bremen, Germany), positioned $30 \mathrm{~cm}$ in front of the patient's head. During nighttime polysomnography, the microphone was placed $30 \mathrm{~cm}$ above the patient's heda. The frequency response of the microphone was $30-20000 \mathrm{~Hz}$. Data were collected by a computer software (Cool Edit 2000, Syntrillium Software Corporation, Phoenix, AZ, USA) at a sampling rate of $44100 \mathrm{~Hz}$. The digitalized snoring sounds were analyzed by fast fourier transformation (FFT) on 16384 samples using a Hanning window[8].

The sound sensor is a unidirectional electret condenser microphone. MK224, considering measurement requirement, its frequency measuring range from $0.1 \mathrm{~Hz}$ to $20 \mathrm{kHz}$, and its sensitivity is $49 \mathrm{mv} / \mathrm{pa}$. Coupled to the skin through a conical air cavity, which is placed laterally on the trachea at the level of the cricoid's cartilage using an elastic band. The etypic signal pulse pass through bandpass filter, only frequency from $100 \mathrm{~Hz}$ to $800 \mathrm{~Hz}$ may pass through and the signal has a high level output after through monostable flip-flop circuit, then the output signal with noise pass through the opto-coupler and its noise are isolated. Light output coupling signals with snoring information processing are received by computer soundcard.

\section{$B$. Simulation of discrete wavelet transform}

According to the sampling theorem of Mallat algorithm[9], if $f_{s}$ is digital signal sampling frequency, the maximum frequency of the signal will be $f_{s} / 2$. if the signal is decomposed by L-level then it will has been divided into $\mathrm{L}+1$ sub-frequency bands, namely:

$$
\left[0, \frac{f_{s}}{2}\right]=\left[0, \frac{f_{s}}{2^{L+1}}\right] \cup\left[\frac{f_{s}}{2^{L+1}}, \frac{f_{s}}{2^{L}}\right] \cup \ldots \cup\left[\frac{f_{s}}{2^{2}}, \frac{f_{s}}{2}\right]
$$

Their corresponding to the sub-band signals are $f_{L}^{a}(n), f_{L}^{d}(n), \ldots f_{2}^{d}(n), f_{1}^{d}(n)$ and there is

$$
f(n)=f_{L}^{a}(n)+\sum_{j-1}^{L} f_{j}^{d}(n)
$$

Data were obtained from 40 subjects with a snoring history. Inclusion criteria were reported snoring (not reported apneas), either by themselves or by the bed partners. The intention of asking for snoring instead of apneas was to obtain a heterogeneous spectrum including different types of snoring, which is not restricted to patients with suspected OSAS. Exclusion criteria was denial of nighttime snoring. The obtained DWT frequency spectra were analyzed in terms of intensity peaks. By applying DWT, a frequency response curve of a specific noise is obtained. The most dominant frequency of a noise is identified by its peak within the frequency response curve. Less prominent frequencies create lower peaks within the response curve. The original snoring events were classified as the benign snore and the typical apneic snore. The spectral peaks for the benign snore and the apneic snore were showed in Fig.1(a) and Fig.1(b) respectively. 


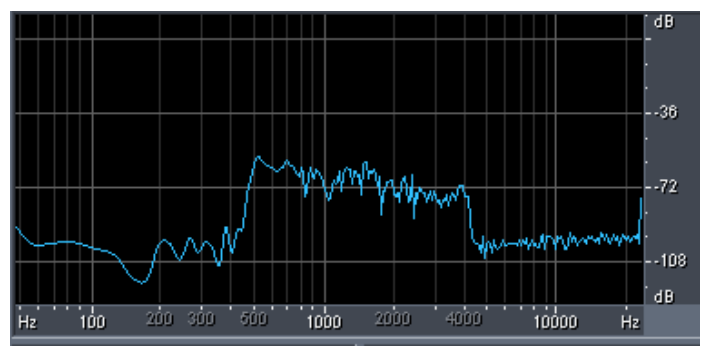

Fig.1(a)

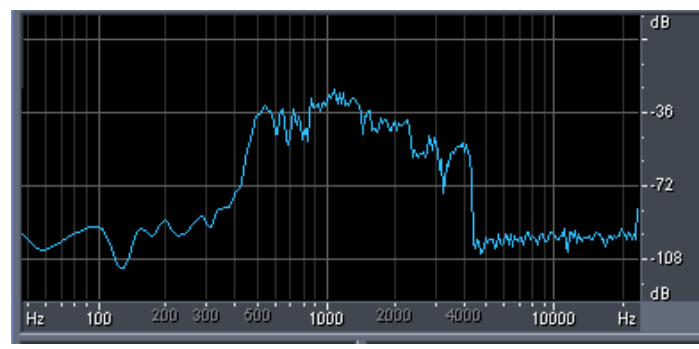

Fig.1(b)

Fig.1 The spectral peaks for the benign snore and the apneic snore

Two examples of frequency analysis in a snoring episode with predominately low intensity peaks and high intensity peaks were demonstrated in the Fig. 1 respectively. In addition to a wider frequency band, the benign snore can exhibit nonlinear couplings in a lower frequency $500 \mathrm{~Hz}$ than that of the apneic one $1200 \mathrm{~Hz}$. It is that the frequency of peaks in the Fig.1(a) is lower than that of peaks in the Fig.1(b). In fact, according to their basic frequencies the patient, Morishima classified adults' snores three types[10]. Type II is that partially unclear due to noise components with variations in basic frequency. Vibrations of the soft palate and uvula contain noise components due to obesity and/or collapse of the tongue base. Type III is strong noise components due to obesity and/or collapse of the tongue base[11]. Analyzing the benign snoring signal frequency distribution, it will be simulated real snoring signals and will be decomposed into the six layers[12]. And the result of the various sub-band benign snoring signals frequency domain analysis are showed in the Fig. 2 and Fig. 3.

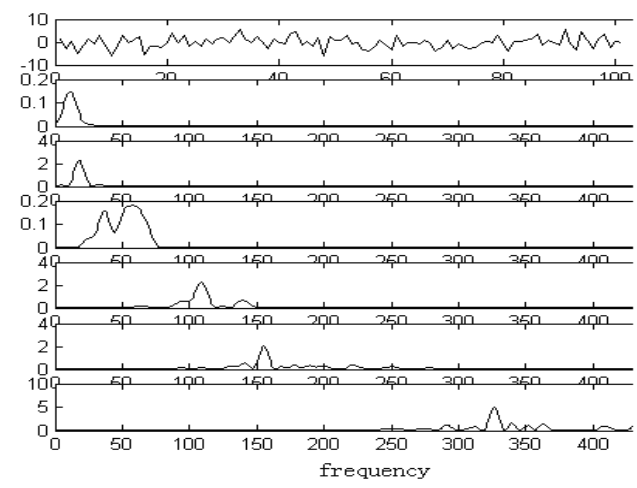

Fig.2 The low-frequency pulse signal spectral characteristics of the subband benign snoring signals

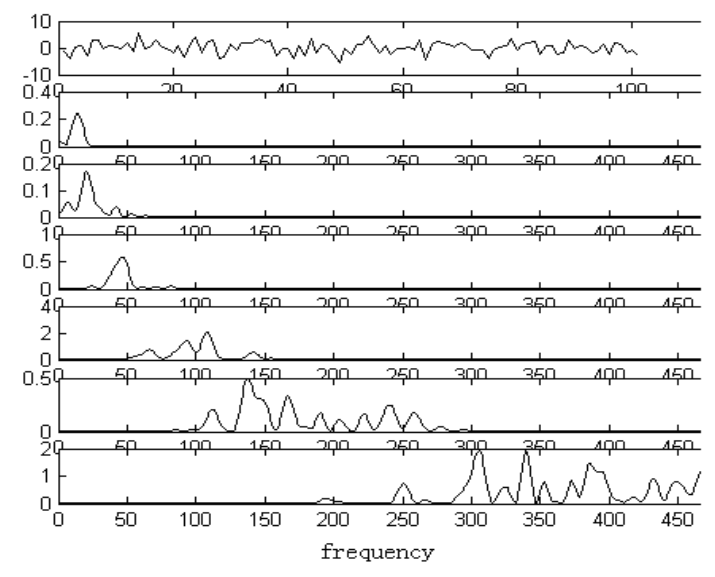

Fig.3 The high-frequency pulse signal spectral characteristics of the sub-band benign snoring signals

\section{Results discussion and clinical diagnosis}

It shows that if the abnormal signal or a pulse interference signal appear, its waveform amplitude will show in an interference pulse, then using a simple frequency-domain criterion, we can accurately deduce them appearing band, where we may extract the frequency of non-rhythmic and rhythmic snoring signal to compare with them. If a certain frequency band signal is filtered, then the respiratory tract will be obstructed. In Fig.2, Low-frequency interference exist in the benign snoring , and more lower frequencies of the snoring signal were filtered, which indicate that the upper breath airway obstruction is not severe. On the contrary, Yet highfrequency pulse signal consist in the more severe snoring signal (Fig.3), and filter the frequency of low frequency signals more than the frequency of high frequency signal, which indicate that the upper breath airway obstruction is serious. The soft palate vibrates at a frequency of $150-500 \mathrm{~Hz}$, during snoring. According to analysis the spectral characteristics of the signal in Fig.2 and Fig.3, the first type of snoring is that involving flutter of the soft palate; the second is that caused by collapse and vibration of the pharyngeal airway. we can realized that it is the potential of the dysfunction in the upper respiratory tract, and which provide a very timely information for medical diagnosis and help us distinguish between benign snoring and malignant snoring which is related to hypoxiablood type of snoring sound in order to exclude sleep apnea syndrome and diagnosis and treatment bronchial asthma during the night.

\section{$V$. conclusion}

Snoring analysis is important to the diagnosis and treatment of the sleep disturbance. It is hoped that study of the biomechanics of snoring will help to produce further advances in treatment in the future. This paper discusses the discrete wavelet transform based on orthogonal signal analysis of snoring sound method, use for wavelet transform snore signal transient pulse to test it. Wavelet transform can have a variety of different frequencies intertwined with the composition of mixed-signal decomposed into different frequency signals, and it can decomposed mixed signal which include various interwoven with different frequency components into different 
frequency signals, and stratified sampling the frequency of different size with corresponding signal in time-space domain, thus it can step into some comprehensive details and vary signal spectrum for analysis. Wavelet transform, it can distinguish between benign snoring and hypoxia-related malignant blood type of snoring and eliminate sleep-apnea syndrome at the night. It provides very timely information for bronchial asthma to medical diagnosis and treatment. Wavelet transform signal analysis method, which is very effective to the non-stationary signal feature analysis. Hence, a wide variety of new future opportunities and ideas might arise from this new approach. The biomedical signal contains lots of different frequency components of the transient information [13], therefore the wavelet transform will have broaden application in biomedical signal processing. Experimental results show that wavelet transform has obvious advantage in the snore sound segment signal analysis and processing and that can detect easily and effectively the signal containing different components. This paper provides an example applying this technique to physiological data. Hope this technique can have broader application in sleep state transition analyses.

\section{Acknowledgment}

This paper is supported by the Fundamental Research Funds for the Central Universities ( Program No. 2010ZYGX020) and the National Natural Science Foundation of China (Grant No.61102094 ).

\section{References}

[1] Cohen, A., "Signal processing methods for upper airway and pulmonary dysfunction diagnosis," IEEE Engineering in Medicine and Biology Magazine, March 1990, 72-75.

[2] Aittokallio, T., Gyllenberg, M., Polo, O., 2001. A model of a Snorer'supper airway. Mathematical Biosciences 170 (1), 79-90.

[3] Daubechies, I. The Wavelet transform time-frequency localization and signal analysis. IEEE Transactions on Information Technology,1990,36(5), 961-1005.

[4] Mallat, S. A theory for multiresolution signal decomposition: The wavelet representation. IEEE Transactions on Pattern Analysis and Machine ntelligence, 1989,11(7), 674-693.

[5] Min Luo, Mohamed Abdelahman and Jeff Frolik. Wavelet-Based Sensor Fusion for Data having Different Sampling Rates. Proceedings of the American Control Conference Arlington, VA June 25-27, 2001.

[6] Purohit A, Bohadana A, Kopferschmitt-Kubler MC et al. Respir Med, 1997, 91:151-157.

[7] Azadeh Yadollahi, Zahra Moussavi. Automatic breath and snore sounds classification from tracheal and ambient sounds recordings. Medical Engineering \& Physics 32( 9), 11, 2010, 985-990.

[8] Michael Herzog et al. Frequency analysis of snoring sounds during simulated and nocturnal snoring. Eur Arch Otorhinolaryngol (2008) 265:1553-1562.

[9] Shi Xizhi. Signal Processing and Soft Computing [M]. Beijing: Higher Education Press, October 2003. 225-237.

[10] N.Morishima, Acoustic analysis of snoring in snorers and sleep apnea patients, Bulletin of the Fujita Medical Society 14(1995) 285-318 (in Japanese).

[11] H.Hattori et al. Acoustic analysis of pediatric snoring. International Congress Series 1257 (2003) 219-225.

[12] Hu Changhua. Analysis and Design based on MATLAB 6.X---- Wavelet Analysis [M]. Xi'an: Xidian University Press, January 2004. 225-237

[13] Yang Fusheng, Gao Kai. Biomedical Signal Processing [M]. Higher Education Press, 1989, 564-588. 